\title{
DYNAMICAL SIMULATIONS: METHODS AND COMPARISONS
}

\author{
D.C. HEGGIE \\ Department of Mathematics and Statistics, University of Edinburgh, \\ King's Buildings, Edinburgh EH9 3JZ, U.K. \\ MIREK GIERSZ \\ N. Copernicus Astronomical Center, Polish Academy of Sciences, \\ ul. Bartycka 18, 00-716 Warsaw, Poland \\ RAINER SPURZEM \\ Astronomisches Rechen-Institut, Moenchhofstrasse 12-14, \\ D-69120 Heidelberg, Germany \\ AND \\ KOJI TAKAHASHI \\ Department of Earth and Space Science, Osaka University \\ Toyonaka, Osaka 560, Japan
}

\section{A Comparative Assessment of Dynamical Models by D.C. Heggie}

\subsection{INTRODUCTION}

In the dynamical study of globular star clusters, five types of dynamical models are in current use. The following list includes recent highlights, some of which are developed in these proceedings by other authors.

1. Static models: Besides the traditional King models and their variants, non-parametric models are of increasing importance (cf. Meylan, these proceedings). Dynamical evolution has no role in static models, however.

2. Scaling models, in which a cluster is assumed to evolve along a simple sequence like the King sequence, which can be characterised in terms of a length and mass scale (and perhaps other parameters, such as the concentration.) These have not been used much since the work of Chernoff \& Shapiro (1987).

3. Gas models: Though little used for the modelling of observations, these models have played a major role in theory, as the phenomena of core collapse and gravothermal oscillations were first developed in this context; cf. Spurzem, this paper, Sect. 4.

4. Fokker-Planck models remain the principal tool for studying the dynamical evolution of both individual clusters (e.g. Sosin \& King 1997) and the cluster system as a whole (e.g. Gnedin \& Ostriker 1997, Murali \& Weinberg 1997). Recent exciting developments are described in Einsel \& Spurzem (1997) and in contributions to this paper by Takahashi (Sect. 2) and by Giersz (Sect. 3).

5. N-body models are increasingly directed towards study of globular clusters (with suitable scaling), thanks to spectacular hardware developments. Along with applications and future developments, these are described in contributions to these proceedings by Makino, Taiji, Vesperini, Tout and Portegies Zwart. 


\subsection{INTERCOMPARISON OF DYNAMICAL MODELS}

All the above kinds of models depend on simplifying assumptions, and their reliability may be investigated by studying the same problems with different models. For example, observations of mass segregation in star clusters are usually interpreted in terms of static, multi-mass King models, and so a comparison of such models with evolved multi-mass Fokker-Planck models is a guide to their reliability. Though some comments on this are to be found in the literature (e.g. Chernoff \& Weinberg 1990, Sosin \& King 1997), a systematic study is overdue.

Comparisons between the three main evolutionary models (gas, Fokker-Planck and $N$-body) are more plentiful (e.g. Aarseth, Hénon \& Wielen 1974, Bettwieser \& Inagaki 1985, Giersz \& Spurzem 1994, Spurzem \& Takahashi 1995, Spurzem \& Aarseth 1996, Theuns 1996). Nevertheless most of these comparisons concern single-mass systems, and almost all deal with isolated clusters.

Because of the interest in a more realistic comparison, in recent months a number of groups have collaborated by working with a variety of codes on a single, somewhat more elaborate model. Initially the model is a non-rotating King model (with $W_{0}=3$ ), with a total mass of $6 \times 10^{4} M_{\odot}$ consisting entirely of single point masses with a Salpeter mass function in the range $0.1<m / M_{\odot}<$ 1.5 and no initial mass segregation. The cluster is in a circular galactic orbit of radius $\simeq 4.2 \mathrm{kpc}$ with a tidal radius of 30pc. Heating is by three-body binaries (which are formed around and after core bounce). No form of stellar evolution is included.

This prescription is a compromise between realism and the limitations of existing codes. In the event, three $N$-body codes, six Fokker-Planck codes and one gas code were able to produce data, thanks to the efforts of S.J. Aarseth, G.A. Drukier, C.R.W. Einsel, K. Engle, T. Fukushige, M. Giersz, D.C. Heggie, P. Hut, H.-M. Lee, J. Makino, S.L.W. McMillan, S.F. Portegies Zwart, G. Quinlan, R. Spurzem and K. Takahashi. Some detailed results can be seen at http://www.maths.ed.ac.uk/people/douglas/experiment.html, and an extended account of the collaboration is in preparation. Here we summarise some points of interest.

All models in the present study indicate core collapse at around 10Gyr and those that reached dissolution did so by around 20Gyr. Fig.1 shows data on the time of core collapse and the total mass at that time (which relates to the rate of mass loss and hence the dissolution time). All the continuum models produce a fairly consistent collapse time, as do the largest $N$-body models. The mean collapse time of the $N$-body models is earlier than that for the continuum models, but most of the discrepancy could be removed by changing the Coulomb logarithm to about $\ln 0.06 N$.

The mass at core bounce is independent of the choice of Coulomb logarithm. All kinds of models show considerable variation, especially the $N$-body models. What is especially interesting about the latter is that the results vary systematically with $N$, in the sense that larger models lose mass more quickly, and that the trend is similar in all three sets of models (which were obtained by independent groups using independently written codes). Also, there is little indication that the results are converging with increasing $N$. The actual number of stars in the cluster which is being simulated is approximately $2.5 \times 10^{5}$, and a rough extrapolation of the $N$-body results with $N$ suggests that the mass at core bounce could be as small as $1.5 \times 10^{4} M_{\odot}$. If so, it follows that existing $N$-body models and all continuum models are in error (for this model) by a factor of about two, in the sense that they lose mass too slowly.

One of the most interesting discoveries from this investigation is the aforementioned trend of the $N$-body models with $N$. No such trend should occur if the evolution scales with the relaxation time, as is assumed by the continuum models. It may be that the Coulomb logarithm must be chosen with greater care: it may involve the number of stars in the core, or the interaction between different pairs of masses may require different values. It may be that there are other processes (related to escape or the anisotropic tidal field) whose time scales do not scale with the relaxation time. Uncovering the reason for the trend with $N$ will require further research. In the meantime, the time scale for cluster evolution obtained from continuum and $N$-body models should be treated with caution.

This work was supported in part by the UK Particle Physics and Astronomy Research Council 


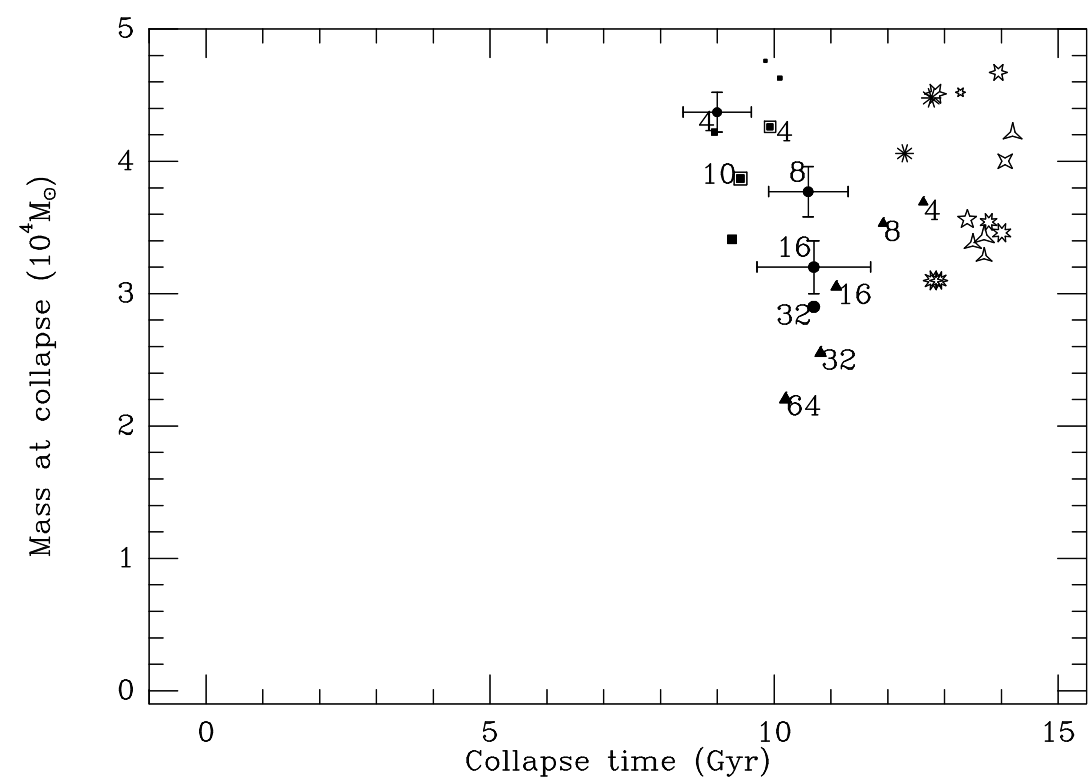

Figure 1. Mass within the tidal radius at the time of core bounce, plotted against the time at which core bounce occurred. The symbols have the following meanings: star - anisotropic gas model; open symbols - Fokker-Planck models; filled symbols $-N$-body models. Symbols of the same shape correspond to results from the same group or individual. Larger symbols denote "better" models, corresponding to larger $N$ (for $N$-body models) or larger numbers of mass groups and/or smaller timesteps (for continuum models). In some cases different symbols correspond to different treatments of the tidal boundary condition; for the $N$-body models with square symbols, the models with a circumscribed rectangle use the same boundary conditions as the $N$-body models denoted by triangles and disks. Most of the latter are obtained from several runs with the same $N$, and the error bars on those cases denote $1 \sigma$. All models have been reduced to a common choice of the Coulomb logarithm $(\ln 0.01 N)$ in the expression for the relaxation time. The value of $N$ is given (in units of 1024) beside the main $N$-body models. The open, three-sided symbols give four results for Takahashi's Fokker-Planck models; the most discrepant (with the highest mass) is an anisotropic model with apocentric escape criterion (cf. his contribution to these proceedings).

under grant GR/J79461; this grant funded GRAPE hardware which was kindly supplied by the group of Prof. D. Sugimoto, University of Tokyo.

\section{Anisotropic Fokker-Planck Models of Globular Cluster Evolution by K. Takahashi}

\subsection{INTRODUCTION}

The dynamical evolution of globular clusters driven by two-body relaxation was investigated by numerical integration of the two-dimensional Fokker-Planck (FP) equation in energy-angular momentum space (Cohn 1979, 1980). The two-dimensional FP models allow anisotropy of the velocity distribution of stars. In this paper, we report the results of our FP simulations of the evolution of globular clusters, and discuss in particular the development of velocity anisotropy and its effects on the cluster evolution.

If spherical symmetry and dynamical equilibrium of a cluster are assumed, then the distribution function $(f)$ is a function of the energy per unit mass $(E)$, the modulus of the angular momentum per unit mass $(J)$, and time $(t)$. If isotropy of the velocity distribution is assumed furthermore, then $f$ is a function of only the energy and time. Anisotropic FP models are, of course, more realistic models of globular clusters than isotropic models.

Numerical integration of the FP equation was performed by using a method described in Takahashi $(1995,1996)$. We considered the mass spectrum of stars (Takahashi 1997) and the effect of tidal truncation (Takahashi et al. 1997) in order to investigate the realistic evolution of globular clusters. 


\subsection{RESULTS}

In isolated single-mass clusters, the halo rapidly becomes dominated by radial orbits. The ratio of the radial velocity dispersion to the tangential one increases monotonically as the radius increases. Also in isolated multi-mass clusters, the radial anisotropy develops in the halo. However, the radial profiles of the velocity anisotropy are significantly different between different masses in some cases. For example, a strong tangential anisotropy can develop around the half-mass radius for massive components in a cluster with a steep mass spectrum. In tidally truncated clusters, although the radial anisotropy develops in the halo during the pre-collapse evolution, the anisotropy becomes highly depressed during the post-collapse evolution due to rapid loss of radial orbits. When the tidal field is weak, the cluster loses mass faster in the anisotropic model than in the isotropic model. However, the difference in mass loss rate between the two models becomes smaller as the strength of the tidal field increases. This is because there is not enough room for the radial anisotropy to develop when the tidal field is strong.

\subsection{DISCUSSION}

We adopted two different tidal-cutoff conditions in $(E, J)$ space: "apocenter condition" and "energy condition" (Takahashi et al. 1997). The apocenter condition removes stars whose apocenter radii are greater than the tidal radius. The energy condition removes stars whose energies are greater than the tidal energy (or the potential energy at the tidal radius). Intuitively the apocenter condition seems to be a more realistic cutoff condition. However, a comparison with $N$-body simulations (cf. Heggie's talk in this session) does not necessarily support this idea. Further investigation is needed on this problem.

This work was supported in part by the Grant-in-Aid for Encouragement of Young Scientists by the Ministry of Education, Science, Sports and Culture of Japan (No. 1338).

\section{Monte-Carlo Simulations. First Results by M. Giersz}

\subsection{INTRODUCTION}

A revision of Stodółkiewicz's Monte-Carlo code (Stodółkiewicz 1982, 1985, 1986) was used to simulate the evolution of star clusters. The Monte-Carlo method can be regarded as a statistical way of solving the Fokker-Planck equation. The great advantages of this method, beside of its simplicity and speed, are connected with the inclusion of anisotropy and with the fact that added realism does not slow it down. The Monte-Carlo method can practically cope as easily as the $N-$ body method with internal degrees of freedom of single and binary stars and external environment, with one exception, a stellar system must be spherically symmetric. The new method treats each superstar as a single star and follows the evolution and motion of all individual stellar objects. This enables, for example, proper description of the densest parts of the system and mass segregation of binaries.

\subsection{FIRST RESULTS}

The first calculations (Giersz 1997) for equal-mass N-body systems with three-body energy generation according to Spitzer's formulae show good agreement with direct $N$-body calculations for $N=2000$, 4096 and 10000 particles. The density, velocity, mass distributions, energy generation, number of binaries etc. follow the $N$-body results. The only difference is that there is no levelling off of the anisotropy level for advanced post-collapse evolution of Monte-Carlo models as is seen in $N$-body simulations for $N \leq 2000$. For simulations with $N \geq 10000$ gravothermal oscillations are clearly visible (see Fig.2). This is the first unambiguous detection of gravothermal oscillations in Monte-Carlo simulations. Moreover, this is a first unambiguous detection of gravothermal oscillations for a stochastic $N$-body system with $N$ as large as 100000 . It should be noted that for 


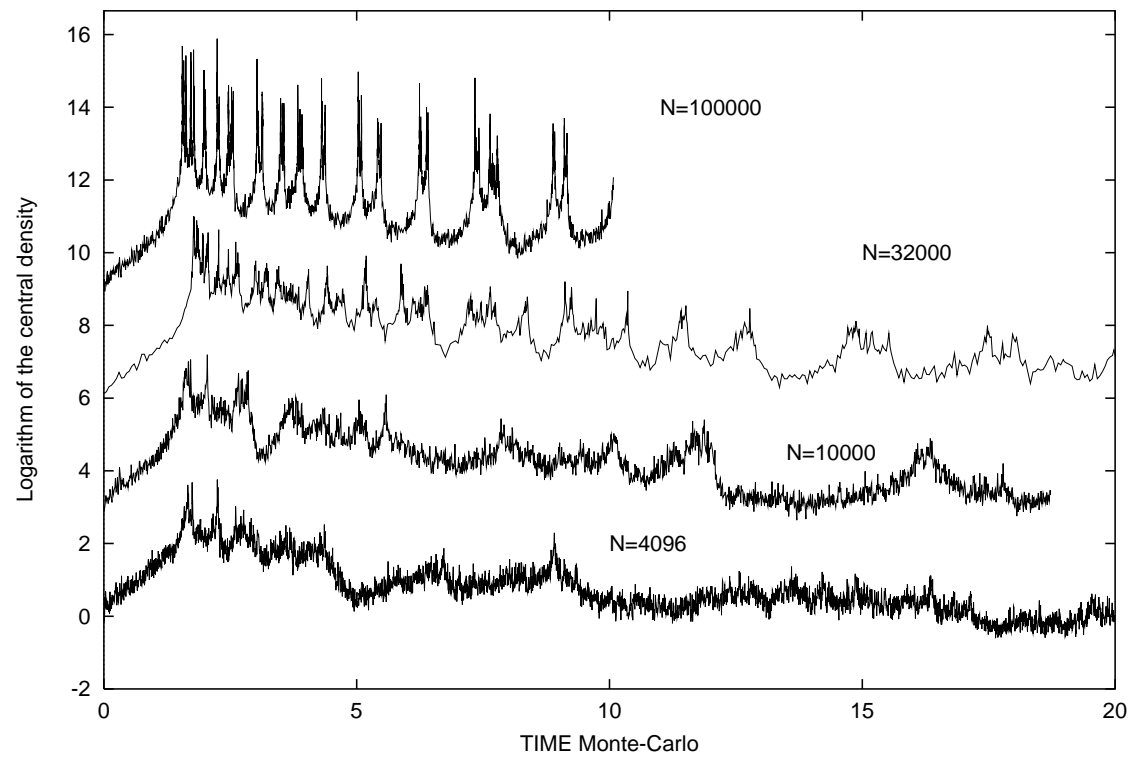

Figure 2. Evolution of the central density for $N=4096,10000,32000$ and 100000. Data are shifted in the logarithm by 3,6 and 9 for $N=10000,32000$ and 100000, respectively.

oscillations observed in Monte-Carlo simulations there is no clear transition from regular oscillations to chaotic ones or from stable expansion to oscillations, as observed in gas and Fokker-Planck models. However, the present results are consistent with results obtained by Takahashi \& Inagaki (1991) for stochastic Fokker-Planck model (stochastic binary formation and energy generation), by Makino (1996) for $N$-body simulations and by Giersz \& Spurzem (1997) for anisotropic gaseous model with fully self-consistent Monte-Carlo treatment of binary population.

The Monte-Carlo code is at least $10^{5}$ times faster than the $N$-body one for $N=32768$ with special-purpose hardware (Makino 1996). Thus it becomes possible to run several different models to improve statistical quality of the data and run individual models with $N$ larger then 100000 . The Monte-Carlo scheme can be regarded as a method which lies in the middle between direct $N$-body and Fokker-Planck models and combines most advantages of both methods.

This work was supported in part by the Polish National Committee for Scientific Research under grant 2-P304-009-06.

\section{Anisotropic Gaseous Models of Star Clusters by R. Spurzem}

Gaseous sphere models of star clusters have been powerful tools to examine the dynamical evolution of star clusters. The physical nature of gravothermal collapse (Lynden-Bell \& Eggleton 1980) and the existence of gravothermal oscillations (Bettwieser \& Sugimoto 1984) were detected using gaseous models. They use a phenomenological heat flux equation in order to close the moment equations of the Boltzmann equation with a Fokker-Planck local collisional term. Anisotropy is taken into account for the second order moments (radial and tangential velocity dispersions, compare Spurzem 1994). It was shown that in pre- and post-collapse for particle numbers ranging from $N=250$ to $N=10000$ there is a very good agreement between results of gaseous models, direct numerical solutions of the orbit-averaged Fokker-Planck equation and direct $N$-body simulations (cf Giersz \& Spurzem 1994, Spurzem \& Aarseth 1996). Similarly comparisons between gaseous and other models of star clusters with two different masses provided results in fair agreement with each other (Spurzem \& Takahashi 1995). 
The previously cited work, however, only examined isolated systems consisting of point masses. Surprisingly, and in contrast to other models, no results have been published recently including a tidal boundary and stellar evolution effects using gaseous models. In the course of an ongoing project for doing that, we provide here the first multi-mass gaseous models including a tidal boundary, for comparison with results of the other methods (Heggie, this paper, Sect. 1). To include a tidal boundary is less straightforward than in Fokker-Planck or $N$-body models. In the $N=10000$ direct $N$-body simulation of Spurzem \& Aarseth (1996) most escapers suffered their last scattering well inside the core. Guided by that we employ the ansatz that at each radius $r$ of a spherical star cluster the mass loss rate by escape across the tidal boundary can be determined by

$$
\frac{\delta \rho}{\delta t}=\frac{k x \rho}{t_{\mathrm{esc}}}
$$

Here $\rho$ is the local stellar mass density, $t_{\text {esc }}=r / v_{\text {esc }}$ the local timescale for escape with the escape speed $v_{\text {esc }}=\sqrt{\Phi_{t}-\Phi}$, using the potential difference between $r$ and the tidal radius $r_{t}$. Assuming a Schwarzschild-Boltzmann distribution $x$ is the fraction of stars located in the escape region of velocity space (for the determination of $x$ it is possible to distinguish between an energy and apocentre criterion for escape as in the anisotropic Fokker-Planck models of Takahashi (this paper, Sect. 2 ), and the actual computation of $x$ is done by approximating a three-dimensional volume integral over an ellipsoidal figure by integrations over a certain combination of volumes with rectangular boundaries). Finally $k$ is the "filling degree" of the escaper region, taking into account that most stars in that region quickly escape. So far we tested single mass systems with $10^{4}$ solar-mass stars and the here presented multi-mass models with $6 \cdot 10^{4}$ solar masses in total. Best agreement was reached by choosing $k=1 \cdot 10^{-7} M / \mathrm{M}_{\odot}$, where $M$ is the total mass of the system. $k$ should be determined by a diffusion equation; this and details of the previously described procedure will be given elsewhere.

Another important improvement of gaseous models is inspired by the revival of Hénon type Monte-Carlo models (Giersz 1997). Star formation is believed to start with a high fraction of socalled primordial binaries, most of which are destroyed in three- and four-body encounters during the dynamical evolution. Such a high amount of binaries cannot be treated by the Fokker-Planck equation anymore. Previous work either was not self-consistent (Hut et al. 1992), or assumed in the single mass model some very preliminary cross sections for close binary-binary encounters (Gao et al. 1991). Direct $N$-body simulations with many binaries suffer from the high computational cost (Aarseth \& Heggie 1992). A new self-consistent model including a stochastic binary component treated by Hénon's Monte-Carlo method, including relaxation (i.e. dynamical friction with the single stars and binary-binary relaxation) and close encounters was proposed and successfully tested (Spurzem \& Giersz 1996). It will provide a self-consistent quasi $N$-body representation for large $N$-body systems with a huge number of binaries for extremely small computational cost. Like in a direct $N$-body simulation three- and four-body encounters can be integrated in regularized coordinates and effects of stellar evolution and collisions could be incorporated in the model, which is subject of future work.

This work was supported in part by DFG (German Science Foundation) grant Sp 345/3-3, 10-1.

\section{References}

Aarseth S.J., Heggie D.C., 1992, MNRAS, 257, 513

Aarseth S.J., Hénon M., Wielen R., 1974, A\&A, 37,183

Bettwieser E., Inagaki S., 1985, MNRAS, 213, 473

Bettwieser E., Sugimoto D., 1984, MNRAS, 208, 493

Chernoff D.F., Shapiro S.L., 1987, ApJ, 322, 113

Chernoff D.F., Weinberg M.D., 1990, ApJ, 351, 121

Cohn, H. 1979, ApJ, 234, 1036.

Cohn, H. 1980, ApJ, 242, 765.

Einsel C.R.W., Spurzem R., 1997, MNRAS, submitted

Gao B., Goodman J., Cohn H., Murphy B., 1991, ApJ, 370, 567 
Giersz M., 1997, MNRAS, submitted

Giersz M., Spurzem R., 1994, MNRAS,269, 241

Giersz M. \& Spurzem R., 1997, in preparation

Gnedin O.Y., Ostriker J.P., 1997, ApJ, 474, 223

Hut P., McMillan S., Romani R.W., 1992, ApJ, 389, 527

Lynden-Bell, D., Eggleton, P.P., 1980, MNRAS, 191, 483

Makino J., 1996 ApJ, 471, 796

Murali C., Weinberg M.D., 1997, MNRAS, 288, 749

Sosin C., King I.R., 1997, AJ, 113, 1328

Spurzem R., 1994, in Pfenniger D. \& Gurzadyan V.G., eds., Ergodic Concepts in Stellar Dynamics, Springer:Berlin, 170

Spurzem R., Takahashi K., 1995, MNRAS, 272, 772

Spurzem R., Aarseth S.J., 1996, MNRAS, 282, 19

Spurzem R., Giersz M., 1996, MNRAS, 283, 805

Stodółkiewicz J.S., 1982, Acta Astr., 32, 63

Stodółkiewicz J.S., 1985, in Goodman J. \& Hut P., eds., Dynamics of Star Clusters, Reidel:Dordrecht, 361

Stodółkiewicz J.S., 1986, Acta Astr., 36, 19

Takahashi, K. 1995, PASJ, 47, 561.

Takahashi, K. 1996, PASJ, 48, 691.

Takahashi, K. 1997, PASJ, 49, in press.

Takahashi K. \& Inagaki S., 1991, PASJ, 47, 561

Takahashi, K., Lee, H.M., Inagaki, S. 1997, MNRAS accepted.

Theuns T., 1996, MNRAS, 279, 827 